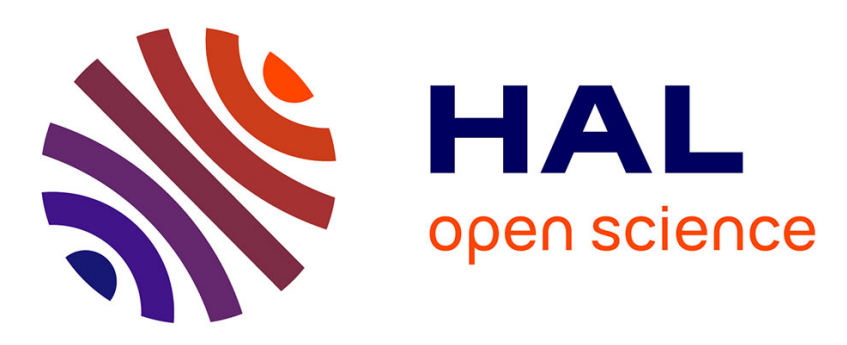

\title{
Design of a passive electrical analogue for piezoelectric damping of a plate
}

Boris Lossouarn, Mathieu Aucejo, Jean-François Deü, Kenneth A. Cunefare

\section{To cite this version:}

Boris Lossouarn, Mathieu Aucejo, Jean-François Deü, Kenneth A. Cunefare. Design of a passive electrical analogue for piezoelectric damping of a plate. Journal of Intelligent Material Systems and Structures, 2018, 29 (7), pp.1301-1314. 10.1177/1045389X17731232 . hal-01739210

\section{HAL Id: hal-01739210 https://hal.science/hal-01739210}

Submitted on 22 Mar 2018

HAL is a multi-disciplinary open access archive for the deposit and dissemination of scientific research documents, whether they are published or not. The documents may come from teaching and research institutions in France or abroad, or from public or private research centers.
L'archive ouverte pluridisciplinaire HAL, est destinée au dépôt et à la diffusion de documents scientifiques de niveau recherche, publiés ou non, émanant des établissements d'enseignement et de recherche français ou étrangers, des laboratoires publics ou privés. 


\title{
Design of a passive electrical analogue for piezoelectric damping of a plate
}

Journal Title

$\mathrm{XX}(\mathrm{X}): 1-17$

(C)The Author(s) 2017

Reprints and permission:

sagepub.co.uk/journalsPermissions.nav

DOI: 10.1177/ToBeAssigned

www.sagepub.com/

@SAGE

\section{Boris Lossouarn", Mathieu Aucejo', Jean-François Deü1, Kenneth A. Cunefare ${ }^{2}$}

\begin{abstract}
Vibrations of a mechanical structure can be reduced through a piezoelectric coupling to a passive electrical network exhibiting similar modal properties. For the control of a plate, the design of a two-dimensional analogous electrical network is considered. Depending on the mechanical boundary conditions, a finite difference formulation of the Kirchhoff-Love equation of motion shows that we need to ensure specific electrical connections along the edges of the analogous network. A numerical model involving an assembly of element matrices validates the electrical topology. Then, the passive electrical circuit is implemented with capacitors, inductors and transformers, whose practical design is closely described. Focusing on the analogue of a clamped plate, experiments prove the ability of the proposed electrical network to approximate the behavior of the mechanical structure.
\end{abstract}

\section{Keywords}

Electrical analogue, Kirchhoff-Love plate, Finite difference method, Multi-resonant network, Modal analysis, Design of magnetic components

\section{Introduction}

A solution for the multimodal control of a plate with a passive piezoelectric network was first proposed by Vidoli and Dell'Isola (2001). Contrary to multi-branch shunts connected to individual piezoelectric transducers (Hollkamp 1994; Wu 1998; Airoldi and Ruzzene 2011), the proposed network consists of inductors that interconnect an array of piezoelectric elements. This allows the synthesis of $2 \mathrm{D}$ electrical modes that match the mechanical modes, both in the spatial and frequency domains, in order to counterbalance the mechanical vibrations. It has been noticed that an optimal eletromechanical coupling can be achieved by considering an electrical network that is the analogue of the structure to control (Dell'Isola et al. 2003; Alessandroni et al. 2002). From this conclusion, research has been conducted on multimodal damping of beams (Porfiri et al. 2004; Andreaus et al. 2004) or plates (Alessandroni et al. 2004, 2005) with passive analogous networks. Although very promising, damping solutions based on analogous piezoelectric networks have not been implemented into any real application. This is most likely due to the fact that no experimental setup had been developed to validate the theoretical concepts. A line of inductors was coupled to an array of piezoelectric patches on a beam (Valis et al. 1994; Dell'Isola et al. 2004) but this cannot generate a real multimodal coupling because it does not involve the electrical analogue of a beam (Porfiri et al. 2004). Then, Panella et al. (2005) decided

\footnotetext{
${ }^{1}$ Structural Mechanics and Coupled Systems Laboratory, Conservatoire national des arts et métiers, France

${ }^{2}$ School of Mechanical Enginering, Georgia Institute of Technology, USA

Corresponding author:

Boris Lossouarn, Structural Mechanics and Coupled Systems Laboratory, Conservatoire national des arts et métiers, 292 Rue SaintMartin, 75003 Paris, France.

Email: boris.lossouarn@cnam.fr
} 
to synthetize such an electrical network with operational amplifiers. Electrical circuits solving stability issues were proposed (Paschero et al. 2006) but it seems that they have not been integrated into a complete piezoelectric array. The use of synthetic components was decided because the passive transformers involved in the electrical analogue are difficult to manufacture for broadband and low frequency applications (Maurini 2005). It was yet proven in the 40's that such analogous electrical network can be implemented with passive components. At that time, no powerful digital computers were available and the analogous electrical networks were used to simulate various mechanical problems (MacNeal 1949). Electrical analogues were first proposed for truss bridges (Bush 1934) or assembly of beam structures (Kron 1944; Carter and Kron 1944). Then, Kron extended the analysis to numerous differential equations as the compressible fluid flow equations, the electromagnetic field equations of Maxwell or the wave equations of Schrödinger (Kron 1945, 1948). Concerning the theory of elasticity, 3D models were introduced (Kron 1948; Barnoski and Freberg 1966) but real implementations of analogous networks were usually restrained to simpler cases involving one- or twodimensional structural members. Simulations involving "analog computers" were performed for beams (MacNeal 1949; Benscoter and MacNeal 1952b), plates (MacNeal 1949; Benscoter and MacNeal 1952a), stiffened shells (MacNeal 1954) and even a whole airplane (MacNeal 1951). We note that this branch of the scientific literature has not been fully exploited since the advent of the modern digital computers in the 60's. Even if it was not their original purpose, the previous references finally represent an important resource when considering the implementation of multimodal damping with a passive piezoelectric network.

In this paper, we focus on novel analogous networks that reproduce the dynamics of Kirchhoff-Love plates. As the resulting electrical circuits are intended for reducing structural vibrations through a periodic array of piezoelectric patches, readers can refer to Lossouarn et al. (2016) for results on plate damping. In comparison, no piezoelectric coupling is investigated in the present paper whose main objective is to give a complete description of the simulation and practical implementation of the proposed analogous networks. The analysis is restricted to rectangular plates with either clamped, simply-supported of free boundary conditions. From the plate theory, those classical boundary conditions are converted in the electrical domain by applying a direct electromechanical analogy on a finite difference formulation of the mechanical problem. The following section thus shows a $2 \mathrm{D}$ electrical unit cell involving capacitors, inductors and transformers with specific electrical boundary conditions. Then, a complete electrical network made of several unit cells is modeled by assembling element matrices into a global system representing the analogue of a dynamic stiffness matrix. Numerical computations show that the proposed electrical network is able to approximate the dynamics of a plate. In the last section, the passive electrical network is implemented for a case focusing on the analogue of a clamped plate. The design of electrical transformers is highlighted because parasitic elements can seriously influence the modal properties of the whole network if they are not chosen correctly. Finally, the analogous network is tested through an experimental modal analysis following the same method as if it was a mechanical structure. Electrical plate-like mode shapes are observed and the correlation with numerical results is evaluated.

\section{Electrical analogue of a square plate unit cell}

A finite difference model of a Kirchhoff-Love plate gives an analogous topology based on passive electrical components. For the sake of completeness, we recall some preliminary results given in Lossouarn et al. (2016). Different plate boundary conditions are then considered, leading to analogous connections along the edges of the electrical network.

\section{Finite difference model of a plate}

According to the Kirchhoff-Love theory, the governing differential equation for a thin plate of Young's modulus 


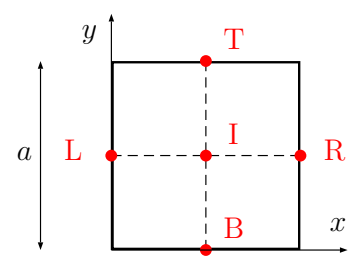

Figure 1. Finite difference grid for a square plate unit cell.

$Y$, density $\rho$, Poisson's ratio $\nu$ and thickness $h$ is given by

$$
-D\left(\frac{\partial^{4} w}{\partial x^{4}}+2 \frac{\partial^{4} w}{\partial x^{2} \partial y^{2}}+\frac{\partial^{4} w}{\partial y^{4}}\right)=\rho h \frac{\partial^{2} w}{\partial t^{2}},
$$

where $D=\frac{Y h^{3}}{12\left(1-\nu^{2}\right)}$ is the bending stiffness, $w=$ $W(x, y) g(t)$ is the transverse displacement, $x$ and $y$ are the two space variables and $t$ is the time variable. Focusing on harmonic motion, $g(t)$ is a trigonometric function satisfying $\partial^{2} g / \partial t^{2}=-\omega^{2} g$. Consequently, the system of equations

$$
\begin{aligned}
& \frac{\partial Q_{x}}{\partial x}+\frac{\partial Q_{y}}{\partial y}=-\rho h a \omega^{2} W \\
& Q_{x}=-\frac{\partial M}{\partial x} \\
& Q_{y}=-\frac{\partial M}{\partial y} \\
& M=a D\left(\frac{\partial \theta_{x}}{\partial x}+\frac{\partial \theta_{y}}{\partial y}\right) \\
& \theta_{x}=\frac{\partial W}{\partial x} \\
& \theta_{y}=\frac{\partial W}{\partial y}
\end{aligned}
$$

is equivalent to equation (1). The constant $a$ corresponds to the side of a square plate unit cell, so that equation (2) is a two-dimensional extension of the state variable system for an Euler-Bernouilli beam (Lossouarn et al. 2015). The variables $Q_{x}$ and $Q_{y}$ represent shear forces and the angles along the principal directions, $\theta_{x}$ and $\theta_{y}$, satisfy the Kirchhoff-Love assumption which states that the normals to the undeformed midplane remain straight after deformation. Yet, the moment variable $M$ appearing in equation (2) does not refer to any classical variable of the plate theory (Timoshenko 1940). It is here introduced for the definition of an adequate finite difference model.

The continuous plate element is discretized by applying the finite difference scheme

$$
\begin{array}{lll}
\left(\frac{\partial[\cdot]}{\partial x}\right)_{\mathrm{L}}=\frac{[\cdot]_{\mathrm{I}}-[\cdot]_{\mathrm{L}}}{a / 2} & \left(\frac{\partial[\cdot]}{\partial y}\right)_{\mathrm{B}}=\frac{[\cdot]_{\mathrm{I}}-[\cdot]_{\mathrm{B}}}{a / 2} \\
\left(\frac{\partial[\cdot]}{\partial x}\right)_{\mathrm{I}}=\frac{[\cdot]_{\mathrm{R}}-[\cdot]_{\mathrm{L}}}{a} & \text { and } & \left(\frac{\partial[\cdot]}{\partial y}\right)_{\mathrm{I}}=\frac{[\cdot]_{\mathrm{T}}-[\cdot]_{\mathrm{B}}}{a} \\
\left(\frac{\partial[\cdot]}{\partial x}\right)_{\mathrm{R}}=\frac{[\cdot]_{\mathrm{R}}-[\cdot]_{\mathrm{I}}}{a / 2} & \left(\frac{\partial[\cdot]}{\partial y}\right)_{\mathrm{T}}=\frac{[\cdot]_{\mathrm{T}}-[\cdot]_{\mathrm{I}}}{a / 2}
\end{array}
$$

where ' $I$ ' is the position at the center of the square unit cell and ' $L$ ', 'R', 'B' and ' $T$ ' refer to the left, right, bottom and top sides, according to the grid in Figure 1. This finite difference approximation is applied to equation (2), which gives

$$
\begin{aligned}
& -Q_{\mathrm{L}}-\left(Q_{\mathrm{B}}-Q_{\mathrm{T}}\right)+Q_{\mathrm{R}}=\mathrm{j} \omega m \dot{W}_{\mathrm{I}} \\
& -M_{\mathrm{L}}+M_{\mathrm{I}}=-\frac{a}{2} Q_{\mathrm{L}} \\
& -M_{\mathrm{I}}+M_{\mathrm{R}}=-\frac{a}{2} Q_{\mathrm{R}} \\
& -M_{\mathrm{B}}+M_{\mathrm{I}}=-\frac{a}{2} Q_{\mathrm{B}} \\
& -M_{\mathrm{I}}+M_{\mathrm{T}}=-\frac{a}{2} Q_{\mathrm{T}} \\
& -M_{\mathrm{I}}=\frac{D}{j \omega}\left(\dot{\theta}_{\mathrm{L}}-\dot{\theta}_{\mathrm{R}}+\dot{\theta}_{\mathrm{B}}-\dot{\theta}_{\mathrm{T}}\right) \\
& \frac{a}{2} \dot{\theta}_{\mathrm{L}}=\dot{W}_{\mathrm{I}}-\dot{W}_{\mathrm{L}} \\
& \frac{a}{2} \dot{\theta}_{\mathrm{R}}=\dot{W}_{\mathrm{R}}-\dot{W}_{\mathrm{I}} \\
& \frac{a}{2} \dot{\theta}_{\mathrm{B}}=\dot{W}_{\mathrm{I}}-\dot{W}_{\mathrm{B}} \\
& \frac{a}{2} \dot{\theta}_{\mathrm{T}}=\dot{W}_{\mathrm{T}}-\dot{W}_{\mathrm{I}}
\end{aligned}
$$

where $m=\rho h a^{2}$ is the mass of the square plate unit cell. This formulation highlights $\dot{W}=\mathrm{j} \omega W$ and $\dot{\theta}=\mathrm{j} \omega \theta$, the derivatives of the transverse and angular displacements that are going to be used as electrical current variables.

\section{Analogous electrical unit cell}

The finite difference model in equation (4) can be converted into its analogous network made of discrete electrical components. Contrary to previous studies on plate electrical analogues (Alessandroni et al. 2005; MacNeal 1949), we focus on the direct electromechanical analogy, which is compatible with the representation of a piezoelectric transducer with passive electrical components (Bloch 1945; Cho et al. 2000; Paganelli et al. 2010). A system involving electromagnetic transducers (Lallart et al. 2008) would rather require dual circuits based on the indirect or forcecurrent electromechanical analogy (Firestone 1933; Bloch 1945; Beranek 1954). Here, as the plate electrical analogue is intended for piezoelectric coupling, we consider the direct analogy, which is based on the equivalence between a voltage and a force $F$ or a moment $M$. Then, we also have an equivalence between an electrical current and a transverse velocity $\dot{W}$ or an angular velocity $\dot{\theta}$.

It becomes possible to represent the system of equations (4) with the electrical circuit given in Figure 2. The electrical transformers of ratio $a / 2$, which are 


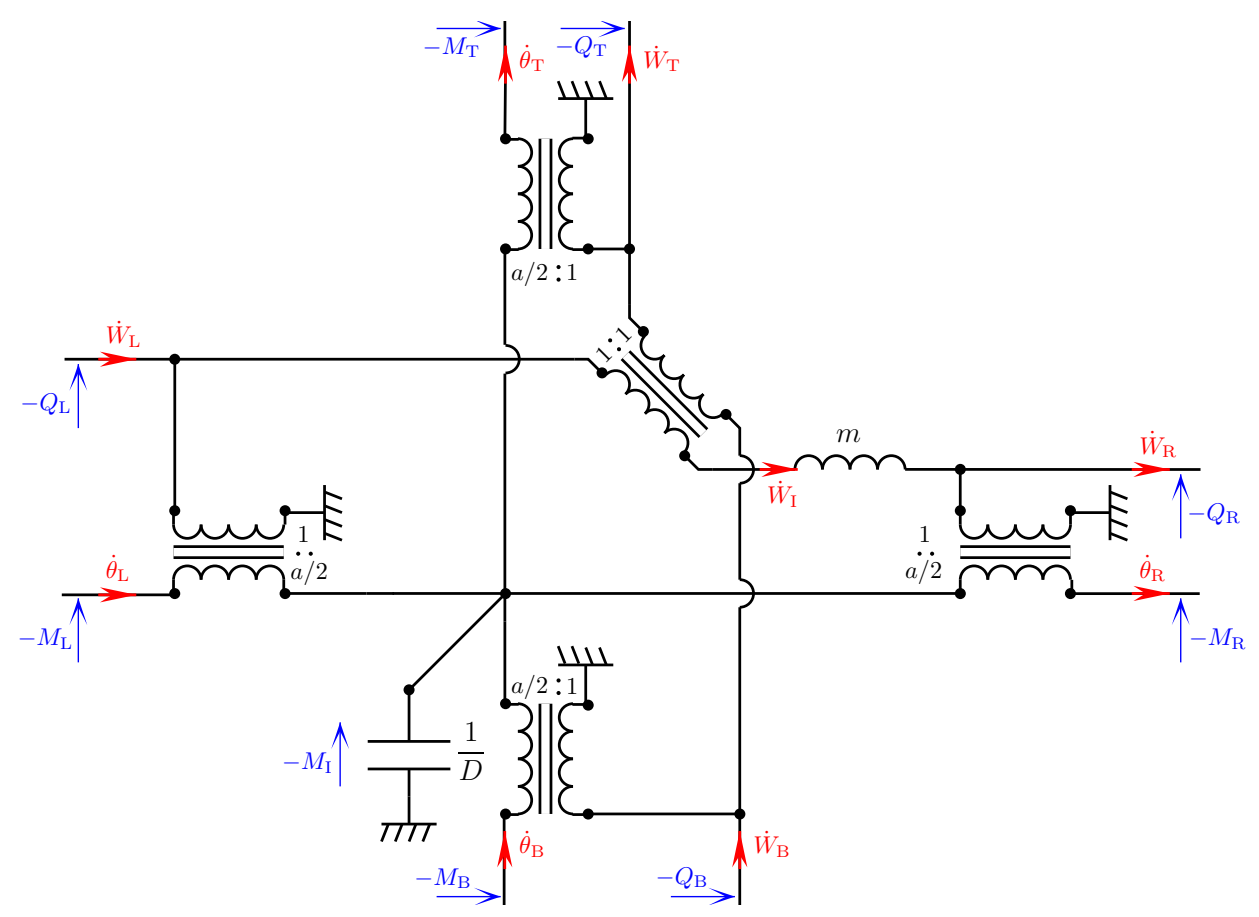

Figure 2. Electrical unit cell as a direct analogue of the plate finite difference model (Lossouarn et al. 2016).

equivalent to mechanical levers of length $a / 2$, allow the implementation of the forward and backward finite differences that satisfy the definition of $\theta$ and $Q$ as spatial derivatives of $w$ and $-M$, respectively. Furthermore, the inductance represents the mass $m$ of the unit cell and the capacitance is equal to the inverse of the bending stiffness $D$, in agreement with the direct electromechanical analogy (Bloch 1945; Beranek 1954). The proposed twodimensional unit cell in Figure 2 can finally be seen as a two-dimensional extension of the beam electrical analogue (Porfiri et al. 2004; Lossouarn et al. 2015). The electrical circuit in Figure 2 has to be replicated $n$ times along the $x$ direction and $m$ times along the $y$ direction in order to create the electrical analogue of a rectangular plate made of $n \times m$ square unit cells. Note that the capacitance $C$ would be replaced by the piezoelectric capacitance when considering electromechanical coupling through an array of piezoelectric patches for a vibration damping purpose (Lossouarn et al. 2016).

A single electrical unit cell is implemented with one inductor, one capacitor and five transformers. It is remarked that the connection of adjacent unit cells reduces the number of components. Indeed, a series of two transformers of ratio $a / 2$ can be replaced by a single transformer with a ratio equal to $a$. Then, the average number of transformers per unit cell tends to three when increasing the number of unit cells. Note that the proposed topology can be seen as a simplification of the "Dynamic analog for a constant thickness plate" introduced by MacNeal in 1949 (MacNeal 1949). This analogous network was obtained from a rectangular plate element and the result is a unit cell made of one capacitor, four inductors and two transformers. It can be shown that the case of the square unit cell simplifies MacNeal's electrical network into the present one, which involves fewer electrical components, i.e. one inductor, one capacitor and three transformers. We also obtain a simpler network when compared to the topology proposed by Alessandroni et al. (Alessandroni et al. 2004, 2005) with one capacitor, three inductors and six transformers per unit cell. As a consequence, the new topology simplifies the practical implementation of the analogous network for experimental validation and application to piezoelectric damping. 


\section{Electrical boundary conditions}

When implementing an analogous electrical network with a set of several unit cells as the one proposed in Figure 2, the electrical states along the edges of the complete network also have to satisfy an analogy with mechanical boundary conditions. For instance, for clamped boundary conditions along an edge at $x=0$,

$$
W(0, y)=0 \quad \text { and } \quad \theta(0, y)=0 .
$$

If this condition applies to the left of the unit cell in Figure 2, we obtain

$$
\dot{W}_{\mathrm{L}}=0 \quad \text { and } \quad \dot{\theta}_{\mathrm{L}}=0,
$$

which means that both electrical port have to remain opencircuited. As explained below, other boundary conditions involving simply-supported edges or free edges are less obvious because they require application of the KirchhoffLove plate theory. The classical state variable formulation of the differential equation in equation (1) can be expressed as follows:

$$
\begin{aligned}
& \frac{\partial Q_{x}}{\partial x}+\frac{\partial Q_{y}}{\partial y}=-\rho h a \omega^{2} W \\
& Q_{x}=\frac{\partial M_{x}}{\partial x}-\frac{\partial M_{x y}}{\partial y} \\
& Q_{y}=\frac{\partial M_{y}}{\partial y}-\frac{\partial M_{x y}}{\partial x} \\
& M_{x}=-a D\left(\frac{\partial^{2} W}{\partial x^{2}}+\nu \frac{\partial^{2} W}{\partial y^{2}}\right) \\
& M_{y}=-a D\left(\frac{\partial^{2} W}{\partial y^{2}}+\nu \frac{\partial^{2} W}{\partial x^{2}}\right) \\
& M_{x y}=a D(1-\nu) \frac{\partial^{2} W}{\partial x \partial y}
\end{aligned}
$$

This corresponds to the variables used by Timoshenko (1940) in which we have introduced the length coefficient $a$ in order to get variables $Q$ and $M$ homogeneous to forces and moments. $Q_{x}$ and $Q_{y}$ are shear forces and their definition is actually the same as in equation (2). The variables $M_{x}, M_{y}$ and $M_{x y}$ are respectively the bending moments along $x$, the bending moment along $y$ and the twisting moment. Simply-supported boundary condition along an edge at $x=0$ corresponds to zero bending moment along $x$ and zero displacement as

$$
W(0, y)=0 \quad \text { and } \quad M_{x}(0, y)=0 .
$$

So, $\left.\quad \frac{\partial^{2} W}{\partial y^{2}}\right|_{0, y}=0 \quad$ and $\quad$ because $\quad M_{x}=$ $-a D\left(\frac{\partial^{2} W}{\partial x^{2}}+\nu \frac{\partial^{2} W}{\partial y^{2}}\right)$, we also get $\left.\frac{\partial^{2} W}{\partial x^{2}}\right|_{0, y}=0$. The variable $M$ involved in equation (2) and in Figure 2 is not equal to the bending moment $M_{x}$ nor $M_{y}$ but it is a linear combination of both of them through

$$
\begin{aligned}
M & =a D\left(\frac{\partial^{2} W}{\partial y^{2}}+\frac{\partial^{2} W}{\partial x^{2}}\right) \\
& =-\frac{1}{1+\nu}\left(M_{x}+M_{y}\right) .
\end{aligned}
$$

With simply-supported boundary conditions, as the second derivatives of $W$ along both $x$ and $y$ directions are equal to zero, $M(0, y)=0$. Consequently, if this applies to the left of the unit cell in Figure 2,

$$
\dot{W}_{\mathrm{L}}=0 \quad \text { and } \quad M_{\mathrm{L}}=0
$$

which corresponds to an open-circuited ' $w$ ' electrical line and a short-circuited ' $\theta$ ' line.

Free boundary condition along the same edge at $x=0$ corresponds to a zero bending moment $M_{x}$ and a zero effective shear force $V_{x}$ :

$$
M_{x}(0, y)=0 \quad \text { and } \quad V_{x}(0, y)=0
$$

where the effective shear force has been defined by Timoshenko (1940) as

$$
\begin{aligned}
V_{x} & =Q_{x}-\frac{\partial M_{x y}}{\partial y} \\
& =-a D\left(\frac{\partial W^{3}}{\partial x^{3}}+(2-\nu) \frac{\partial^{3} W}{\partial x \partial y^{2}}\right) .
\end{aligned}
$$

The boundary condition $V_{x}(0, y)=0$ thus gives

$$
\begin{aligned}
Q_{x}(0, y) & =\left.a D(1-\nu) \frac{\partial^{3} W}{\partial x \partial y^{2}}\right|_{0, y} \\
& =\left.a D(1-\nu) \frac{\partial^{2} \theta_{x}}{\partial y^{2}}\right|_{0, y} .
\end{aligned}
$$

Furthermore, the boundary condition $M_{x}(0, y)=0$ leads to $\left.\frac{\partial^{2} W}{\partial x^{2}}\right|_{0, y}=-\left.\nu \frac{\partial^{2} W}{\partial y^{2}}\right|_{0, y}$, so

$$
M(0, y)=\left.a D(1-\nu) \frac{\partial^{2} W}{\partial y^{2}}\right|_{0, y} .
$$


Finally, with the finite difference scheme, equations (13) and (14) are equivalent to

$$
\begin{aligned}
& Q_{\mathrm{L}}=\frac{D(1-\nu)}{a}\left(\theta_{\mathrm{L}}^{+}-2 \theta_{\mathrm{L}}+\theta_{\mathrm{L}}^{-}\right) \\
& M_{\mathrm{L}}=\frac{D(1-\nu)}{a}\left(W_{\mathrm{L}}^{+}-2 W_{\mathrm{L}}+W_{\mathrm{L}}^{-}\right)
\end{aligned},
$$

where the superscripts ' + ' and '-' refer to the neighboring unit cells along the $x$ axis. We note that $Q_{\mathrm{L}}$, which is the analogue of a voltage in the ' $w$ ' electrical line, is a linear combination of charge displacements occurring in three distinct ' $\theta$ ' electrical lines. A similar situation occurs with $M_{\mathrm{L}}$, voltage in the ' $\theta$ ' electrical line, which is a linear combination of charge displacements occurring in three distinct ' $w$ ' lines. Consequently, even if the boundary conditions in equation (15) can be implemented with an active control system, it seems that it cannot be achieved with simple electrical connections when considering the circuit in Figure 2. This highlights a limit of the proposed network topology, which allows an easy implementation of clamped or simply-supported boundary conditions, but no passive solution for free edges. Investigations related to other boundary conditions or plate geometries could be realized using a similar methodology but it would certainly lead to more complex analogous networks which are not in the scope of the present paper.

\section{Numerical validation of the plate electrical analogue}

A discretized plate or its analogous electrical network are modeled by assembling element matrices. Eigenmodes and frequency response functions are computed for various number of unit cells. This leads to a numerical validation of the proposed electrical analogue.

\section{Element "mass" and "stiffness" matrices}

The electrical circuit in Figure 2 defines a single unit cell of the proposed analogous network, which represents the finite difference model of a square plate unit cell. In order to model a complete electrical network or a complete plate made of several unit cell, it is decided to focus on an assembly of element matrices, as classically performed through finite element procedures. By considering a force and a displacement vector,

$$
\begin{aligned}
& \boldsymbol{F}_{\boldsymbol{m}}^{\mathrm{e}}=\left[\begin{array}{lllllll}
-Q_{\mathrm{B}}-M_{\mathrm{B}} & -Q_{\mathrm{L}}-M_{\mathrm{L}} & Q_{\mathrm{R}} & M_{\mathrm{R}} & Q_{\mathrm{T}} & M_{\mathrm{T}}
\end{array}\right]^{T}, \\
& \boldsymbol{q}_{\boldsymbol{m}}^{\mathrm{e}}=\left[\begin{array}{llllllll}
W_{\mathrm{B}} & \theta_{\mathrm{B}} & W_{\mathrm{L}} & \theta_{\mathrm{L}} & W_{\mathrm{R}} & \theta_{\mathrm{R}} & W_{\mathrm{T}} & \theta_{\mathrm{T}}
\end{array}\right]^{T},
\end{aligned}
$$

one might want to define an element dynamic stiffness matrix $D_{m}^{\mathrm{e}}$ as

$$
F_{m}^{\mathrm{e}}=D_{m}^{\mathrm{e}} q_{m}^{\mathrm{e}}
$$

The signs in the force vector are chosen in order to involve external forces applied to the unit cell. As, $Q_{\mathrm{B}}, M_{\mathrm{B}}$, $Q_{\mathrm{L}}$ and $M_{\mathrm{L}}$ represent mechanical actions applied by the considered unit cell to its bottom or left neighbors, their opposite values are introduced into the force vector. The calculation of the dynamic stiffness matrix $D_{m}^{\mathrm{e}}$ relies on the set of equations (4), which has to be reorganized to make appear the force components as linear combinations of the displacements. Yet, we remark that the displacements are not independent variables. Indeed, equation (4) shows that if all four angular velocities have prescribed values, the choice of a single transverse velocity enforces the value of the other ones. This means that the dynamic stiffness matrix cannot be defined. Additional degrees of freedom are required to relax the kinematic constraints linking angular and transverse velocities. To this end, additional components are inserted in the electrical circuit representing the plate unit cell. The modified circuit is represented in Figure 3, where capacitors $C_{0} / 2$ are added at the ends of the ' $\theta$ ' electrical lines in order to allow the computation of the dynamic stiffness matrix $D_{m}^{\mathrm{e}}$. Additional inductors $L_{\theta} / 2$ are also introduced in the ' $\theta$ ' electrical lines. Those inductors are actually the analogues of moments of inertia. Moreover, they can be used to model parasitic elements in non-ideal transformers when considering the analysis of the analogous electrical network.

Electrical variables have been used in Figure 3 to show that the analysis of an electrical network can reproduce methods usually implemented for the analysis of mechanical structures. Indeed, one can define an electrical "dynamic stiffness matrix" $D_{e}^{\mathrm{e}}$, which satisfies

$$
F_{e}^{\mathrm{e}}=D_{e}^{\mathrm{e}} q_{e}^{\mathrm{e}}
$$




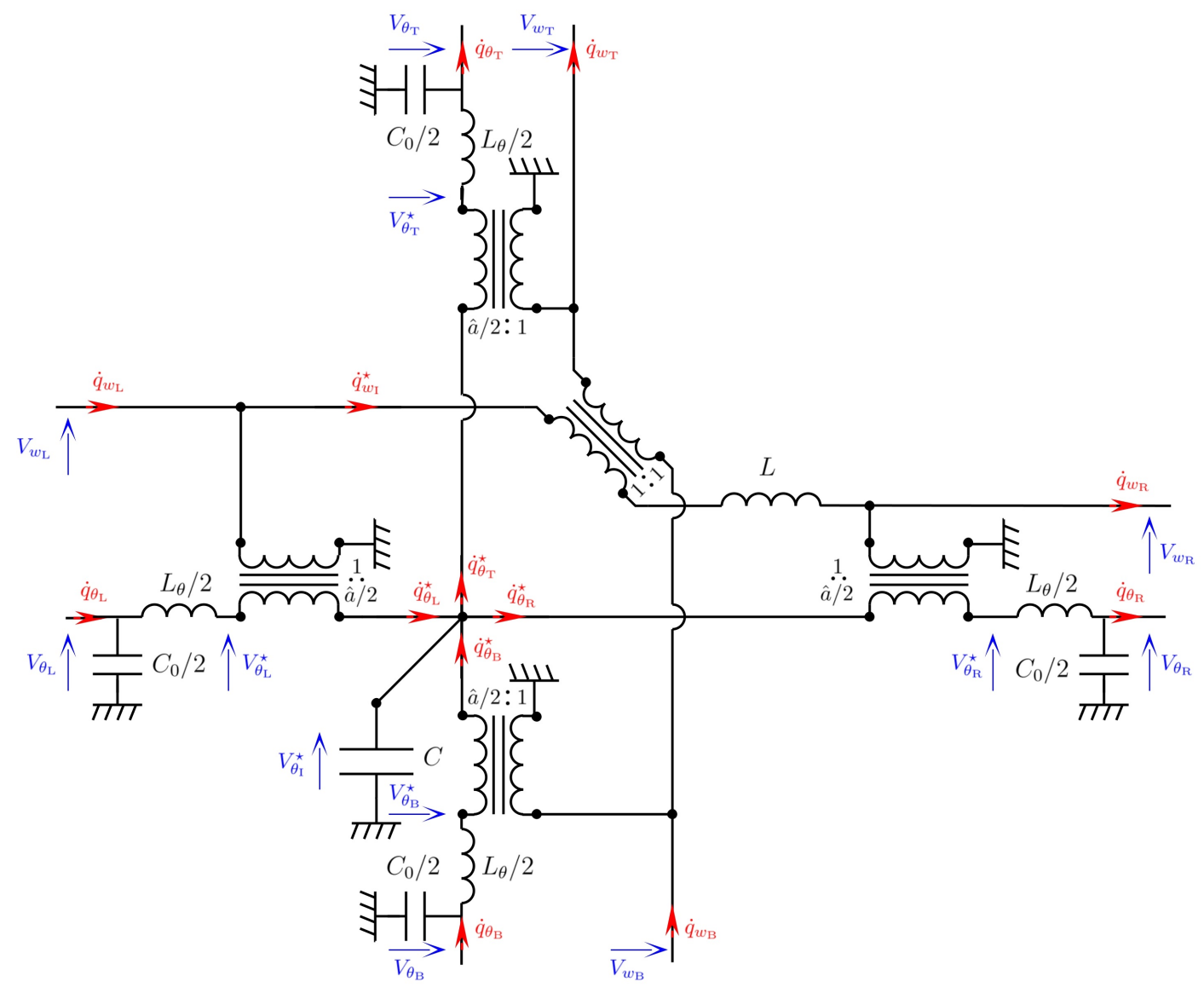

Figure 3. Modified unit cell for the calculation of the element dynamic stiffness matrix.

where

$$
\begin{aligned}
& \boldsymbol{F}_{\boldsymbol{e}}^{\mathrm{e}}=\left[V_{w \mathrm{~B}} V_{\theta \mathrm{B}} V_{w \mathrm{~L}} V_{\theta \mathrm{L}}-V_{w \mathrm{R}}-V_{\theta \mathrm{R}}-V_{w \mathrm{~T}}-V_{\theta \mathrm{T}}\right]^{T} \\
& \boldsymbol{q}_{\boldsymbol{e}}^{\mathrm{e}}=\left[\begin{array}{llllllll}
q_{w \mathrm{~B}} & q_{\theta_{\mathrm{B}}} & q_{w \mathrm{~L}} & q_{\theta_{\mathrm{L}}} & q_{w \mathrm{R}} & q_{\theta \mathrm{R}} & q_{w \mathrm{~T}} & q_{\theta \mathrm{T}}
\end{array}\right]^{T}
\end{aligned}
$$

are the voltage vector and the electrical charge displacement vector, the analogues of the force and displacement vectors, $\boldsymbol{F}_{\boldsymbol{m}}^{\mathrm{e}}$ and $\boldsymbol{q}_{\boldsymbol{m}}^{\mathrm{e}}$. Note that the signs in $\boldsymbol{F}_{e}^{\mathrm{e}}$ are the opposites of the signs in $\boldsymbol{F}_{\boldsymbol{m}}^{\mathrm{e}}$ because the voltages are analogous to the opposite of the force contributions, as shown in Figure 2. The "dynamic stiffness matrix" $D_{e}^{\mathrm{e}}$ is obtained by writing down all the discrete electrical equations referring to Figure 3, eliminating the internal variables and solving for the side voltage variables. Element "stiffness" and "mass" matrices are then defined through

$$
\boldsymbol{D}_{\boldsymbol{e}}^{\mathrm{e}}=\boldsymbol{K}_{\boldsymbol{e}}^{\mathrm{e}}-\omega^{2} \boldsymbol{M}_{\boldsymbol{e}}^{\mathrm{e}}
$$

which means that $\boldsymbol{K}_{e}^{\mathrm{e}}$ corresponds to a "static stiffness matrix":

$$
\boldsymbol{K}_{\boldsymbol{e}}^{\mathrm{e}}=\boldsymbol{D}_{\boldsymbol{e}}^{\mathrm{e}} \quad \text { when } \quad \omega=0 .
$$

So, by setting $\omega=0$ in the symbolic expression for $D_{e}^{\mathrm{e}}$ we obtain the matrix $\boldsymbol{K}_{e}^{\mathrm{e}}$ which is given in Appendix. The capacitance $C_{0}$ has only been introduced to allow the calculation of $\boldsymbol{K}_{\boldsymbol{e}}^{\mathrm{e}}$. It is a numerical parameter which does not influence the results as long as it is small compared to $C$. On the other hand, it needs to be high enough to avoid numerical issues. A value of $C_{0}$ around $C \times 10^{-6}$ was found to be adequate for the following computations.

Then, it still remains to define the element "mass matrix" $M_{e}^{\mathrm{e}}$. Equation (20) gives

$$
\boldsymbol{M}_{\boldsymbol{e}}^{\mathrm{e}}=\frac{\boldsymbol{K}_{\boldsymbol{e}}^{\mathrm{e}}-\boldsymbol{D}_{\boldsymbol{e}}^{\mathrm{e}}}{\omega^{2}} .
$$


Contrary to $K_{e}^{\mathrm{e}}$, the matrix $M_{e}^{\mathrm{e}}$ can be defined in the case of $C_{0}=0$ and it is found that

$$
M_{e}^{\mathrm{e}}=M_{e}^{L}+M_{e}^{L_{\theta}},
$$

where $M_{e}^{L}$ and $M_{e}^{L_{\theta}}$ are given in Appendix. The electrical "dynamic stiffness matrix" in equation (20) is computed with the values of the electrical parameters: $L, L_{\theta}, C$ and $\hat{a}$. The exact same mass and stiffness matrices can be used when considering a mechanical plate element referring to equation (17). This simply requires to replace the inductance $L$ by the mass $m$ of the unit cell, the capacitance $C$ by the inverse of the bending stiffness $D$ and the transformer ratio $\hat{a}$ by the length $a$ of the unit cell.

\section{Modal analysis}

The plate electrical analogue can be modeled by replicating the unit cell in Figure 3 along the $x$ and $y$ directions. Following an assembly process, as in the finite element method, a global stiffness matrix $\boldsymbol{K}$ and a global mass matrix $M$ are built from the element matrices given in Appendix. From these global matrices, it becomes possible to perform a modal analysis by solving the generalized eigenvalue problem

$$
\left[\boldsymbol{K}-\omega^{2} \boldsymbol{M}\right] \boldsymbol{q}=\mathbf{0} .
$$

In the following, we compute the modes of the electrical analogue of a mechanical plate without rotational inertia, i.e. $L_{\theta}=0$. The other electrical constants have to satisfy

$$
\frac{1}{\hat{a}^{2}} \frac{1}{L C}=\frac{1}{a^{2}} \frac{D}{m}
$$

in order to ensure identical modal properties for the electrical network and the corresponding plate finite difference model (Lossouarn et al. 2016).

A modal analysis is performed in order to validate the proposed analogous electrical network. We consider the analogue of a plate of side $l_{x}=400 \mathrm{~mm}$, width $l_{y}=320$ $\mathrm{mm}$ and thickness $h=1.9 \mathrm{~mm}$. The Young's modulus of the plate is $Y=69 \mathrm{GPa}$, its Poisson's ratio is $\nu=0.33$ and its density is $\rho=2700 \mathrm{~kg} / \mathrm{m}^{3}$. In the present section, simply-supported boundary conditions are taken into

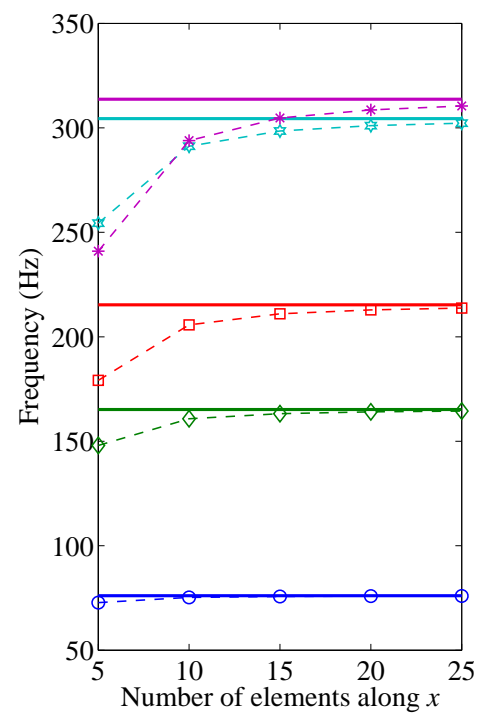

Figure 4. Modal analysis of the proposed electrical analogue and convergence toward the continuous plate model.

account to study the convergence of the discrete electrical network. Indeed, with those boundary conditions the natural frequencies and the mode shapes of a thin homogeneous plate are known analytically (Timoshenko 1940). The theoretical natural frequencies of the first five modes of the plate are represented by horizontal continuous lines in Figure 4. Then, the markers correspond to the natural frequencies computed for the analogous network with either $5 \times 4,10 \times 8,15 \times 12,20 \times 16$ or $25 \times 20$ unit cells. It is observed that the natural frequencies of the discrete network approach those of the continuous plate when increasing the number of elements. Furthermore, the computed mode shapes also give a suitable approximation of the theoretical trigonometric modes of a simply supported plate, as seen in Figure 5 with $25 \times 20$ unit cells. As could have been expected, the highest modes require a larger number of unit cell for a similar precision on the eigenvalues. The following recommendation can be formulated from the numerical results: at least 10 unit cells per wavelength are required to obtain a suitable approximation of a continuous plate. This corresponds to $15 \times 12$ unit cells for the present example focusing on the first five modes of a simplysupported plate. Figure 4 shows that $15 \times 12$ unit cells offer a frequency error of less than $3 \%$. Finally, this modal analysis 


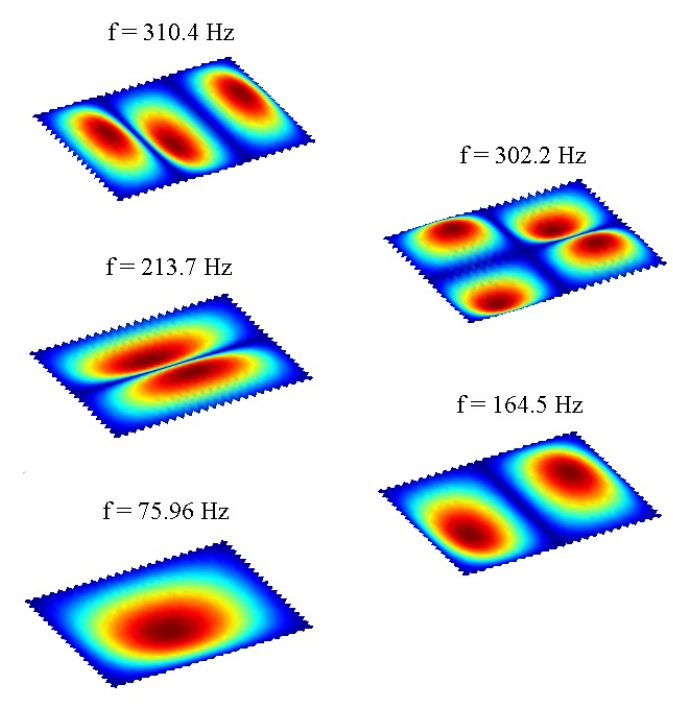

Figure 5. Electrical mode shapes in terms of distribution of the electrical current computed with $25 \times 20$ unit cells.

validates the topology of the proposed analogous electrical network.

\section{Frequency response functions}

The model of the electrical analogue also allows the computation of frequency response functions from

$$
\left[\boldsymbol{K}-\omega^{2} \boldsymbol{M}\right] \boldsymbol{q}=\boldsymbol{F},
$$

where the "force" vector $\boldsymbol{F}$ contains terms representing harmonic excitation of the network. For a mechanical model, if a transverse force $F_{\text {sim }}$ is applied to the left of the unit cell $(i, j)$, i.e. the $i^{\text {th }}$ unit cell along the $x$ axis and the $j^{\text {th }}$ unit cell along the $y$ axis, then

$$
F_{\text {sim }}=Q_{\mathrm{R}}^{(i-1, j)}-Q_{\mathrm{L}}^{(i, j)}
$$

is the term that would appear in the force vector $\boldsymbol{F}$. By analogy, the excitation of an electrical network corresponds to a voltage

$$
V_{\mathrm{sim}}=-V_{w_{\mathrm{R}}}^{(i-1, j)}+V_{w_{\mathrm{L}}}^{(i, j)}
$$

Figure 3 indicates that this excitation is a voltage applied between two transformers in the ' $w$ ' electrical line. This is shown in Figure 6 without representing the capacitors $C_{0} / 2$ that does not appear in the physical circuit. For a

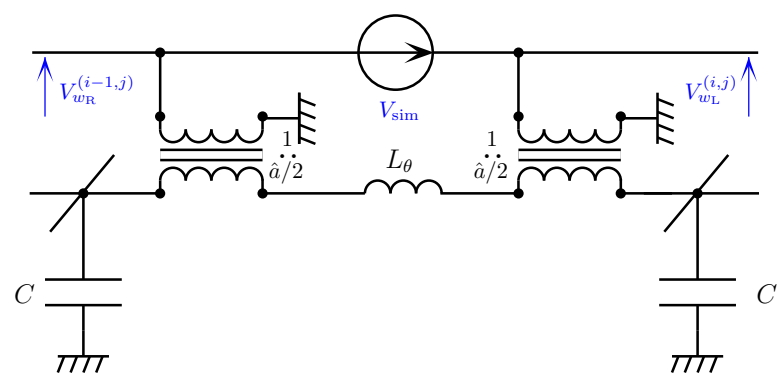

Figure 6. Voltage excitation applied between the unit cells $(i-1, j)$ and $(i, j)$.

frequency analysis of an electrical network, a scalar $V_{\text {sim }}$ is thus inserted in the vector $\boldsymbol{F}$ to simulate the voltage excitation appearing in Figure 6. This is how we proceed to compute electrical frequency response functions in the following comparison with experimental results.

\section{Experiments with a passive analogous network}

The analogue of a thin plate is assembled with passive electrical components whose design is closely described. By analogy with modal vibration analysis of a mechanical structure, the network is excited with a voltage and electrical distributions of the current are measured. The topology of the network is validated by observing "electrical mode shapes" that are equivalent to what would be observed with the analogous mechanical structure.

\section{Implementation of the electrical network}

In order to validate the electrical topology, the analogue of a plate is built by connecting together $5 \times 4$ identical unit cells. This coarse mesh was chosen for practical reasons related to the number of electrical components. Even for the second mode, the resulting network cannot offer a minimum of 10 unit cells per wavelength as suggested previously. There is thus no reason to expect an accurate correlation between the electrical resonance frequencies and those of a continuous plate but we can still hope for distributions of the electrical current that properly reproduce plate-like mode shapes. Furthermore, experiments allow the validation of the proposed numerical model. In the present section, we focus on the analogue 

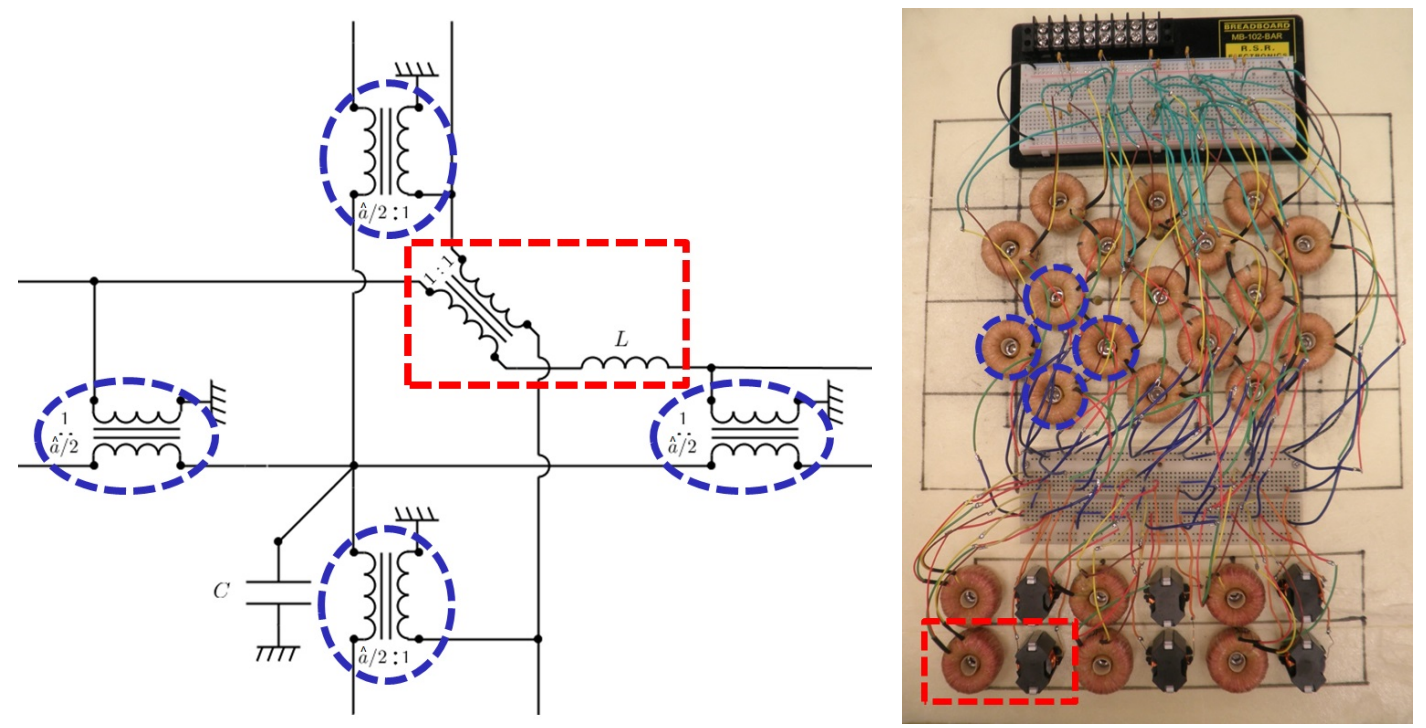

Figure 7. Topology of the unit cell and electrical network made of $5 \times 4$ unit cells: the round frames refer to the transformers on the sides of a unit cell and the rectangular frame refers to the 1:1 transformer and the corresponding inductor.

of a clamped plate so the zero-displacement and zeroangle boundary conditions along the four edges of the plate are implemented by keeping the electrical ports open at the boundaries of the network. As a consequence, no current flows through the inductors and transformers along the four edges of the network, which means that those components are not required and can be removed from the network. For the same reason related to the choice of the boundary conditions, the capacitors at the four corners of the network are not necessary. A last simplification concerns the pairs of $\hat{a} / 2$ ratio transformers, which can be replaced by single transformers of ratio $\hat{a}$. In the end, the network is assembled with 16 capacitors $C=470 \mathrm{nF}$, 17 transformers of ratio 4:1, 6 transformers of ratio 1:1 and 6 inductors $L=0.9 \mathrm{H}$, whose values were selected in view of the final application involving piezoelectric coupling to a clamped plate (Lossouarn et al. 2016). The positioning of the electrical components is shown in Figure 7 , where we note frames with dashed lines that indicate the correspondence between the topology of the unit cell and the actual electrical network.

\section{Design of magnetic components}

The passive electrical analogue of a plate involves ideal transformers. While such components have been

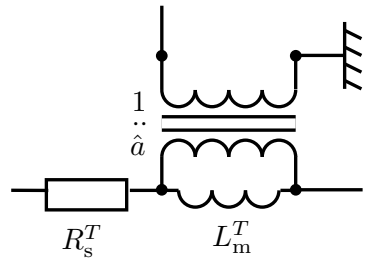

Figure 8. Equivalent circuit of the transformer of ratio $\hat{a}: 1$.

represented in the electrical unit cell in Figure 7, real magnetic transformers are usually far from ideal because of the parasitic effects related to their practical design. In the present case, it has been remarked that no leakage inductance needs to be modeled but the magnetizing inductance $L_{\mathrm{m}}^{T}$ and the winding resistance $R_{\mathrm{s}}^{T}$ cannot be neglected. Those additional elements are seen in Figure 8 for the $\hat{a}: 1$ transformer resulting from the connection in series of two $\hat{a} / 2: 1$ transformers. The same electrical model is considered for the 1:1 transformer in Figure 9, where the magnetizing inductance and winding resistance are respectively $L_{\mathrm{m}}^{T^{\star}}$ and $R_{\mathrm{s}}^{T^{\star}}$. Note that the winding where we have placed the $R_{\mathrm{s}}^{T^{\star}}$ resistance is the one connected to the inductor. Moreover, the damping in the inductor can be modeled by a series resistance $R_{\mathrm{s}}^{L}$ and a parallel resistance $R_{\mathrm{p}}^{L}$. 


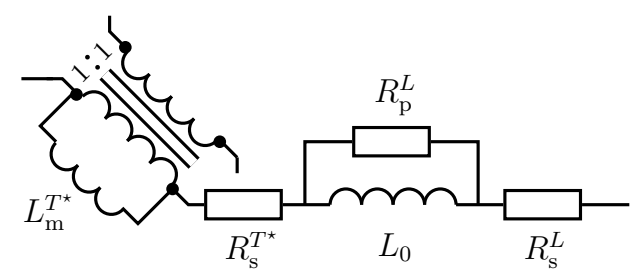

Figure 9. Equivalent circuits of the inductor and the 1:1 transformer.

The inductors are made by winding copper wire around a RM10 magnetic circuit in T38 material that offers a permeance $A_{L}^{L}=16 \mu \mathrm{H}$. We thus need $\sqrt{L / A_{L}^{L}}=$ 237 turns to generate an inductance $L=0.9 \mathrm{H}$. The series resistance is $R_{\mathrm{s}}^{L}=2.6 \Omega$ with a copper wire diameter equal to $0.32 \mathrm{~mm}$ and the parallel resistance of the inductors is then evaluated experimentally to $R_{\mathrm{p}}^{L}=200 \mathrm{k} \Omega$. Concerning the design of the transformers, a sufficient magnetizing inductance is required to approximate an ideal transformer with a magnetic component. It has been remarked that the magnetizing inductance of the secondary winding (in the 'w' electrical line) does not significantly influence the electrical response if it is at least around 50 times larger than the inductance $L$. This means that

$$
L_{\mathrm{m}}^{T} \geq 50 \hat{a}^{2} L
$$

where $L_{\mathrm{m}}^{T}=A_{L}^{T} N_{1}^{2}$ is the magnetizing inductance of the primary winding (in the ' $\theta$ ' electrical line), $A_{L}^{T}$ is the permeance of the magnetic core and $N_{1}$ is the number of turns of the primary winding. With a transformer ratio $\hat{a}=$ 4, equation (29) gives $L_{\mathrm{m}}^{T} \geq 720 \mathrm{H}$. This high inductance value can be satisfied with a $30 \times 20 \times 10$ nanocrystalline toroid in Vitroperm 500F offering a permeance $A_{L}^{T}$ around $100 \mu \mathrm{H}$. After winding $N_{1}=3000$ turns for the primary and $N_{2}=750$ turns for the secondary, a magnetizing inductance $L_{\mathrm{m}}^{T} / \hat{a}^{2}=57 \mathrm{H}$ is measured at $100 \mathrm{~Hz}$ and $1 \mathrm{~V}$ on the secondary winding. This means that $L_{\mathrm{m}}^{T}=$ $912 \mathrm{H}$, which satisfies equation (29). Wires of diameters $0.15 \mathrm{~mm}$ and $0.22 \mathrm{~mm}$ are used for the primary and secondary windings, leading to a total winding resistance $R_{\mathrm{s}}^{T}=353 \Omega$. At last, the 1:1 transformers also need to offer a magnetizing inductance $L_{\mathrm{m}}^{T^{\star}}$ above 50 times the inductance $L$. This condition is satisfied with two windings of 750 turns on the same nanocrystalline toroid as for the 4:1 transformers. A winding resistance $R_{\mathrm{s}}^{T^{\star}}=21 \Omega$ is then obtained with a wire of $0.25 \mathrm{~mm}$ diameter. As will be seen in the following, the winding resistance of the transformers is not negligible, which restrains the amplitude of the electrical resonances.

\section{Experimental setup}

As the proposed electrical network represents the analogue of a plate, it has been decided to validate the electrical behavior with classical instrumentation normally dedicated to mechanical modal analysis. The setup is represented in Figure 10 where a clamped plate (Lossouarn et al. 2016) and an electrical analogue appear together in order to show the analogy in terms of data acquisition. If the considered structure is the mechanical plate, we acquire the input force and the velocity, which is scanned on several points with a vibrometer. If the electrical network is analyzed, the signals of interest are not force and velocity but their direct analogues: the input voltage and the current flowing through the inductors. The direct measurement of the current would require the introduction of instruments in the network, but this could modify its electrical properties. A less intrusive solution consists in measuring the voltage drop across the inductors, which is finally the analogue of the acceleration. This differential voltage is measured by using a low noise preamplifier offering a differential function. The resulting signal and the voltage input are sent to the workstation, where the electrical frequency response function is computed. Spatial distributions of the electrical variables can be obtained by scanning several points of the electrical network. This requires the use of two preamplifiers and a switch, which allows changing the measurement location without any delay. While the voltage drop is measured on one inductor, we have time to prepare the electrical connections for the next measurement. This is crucial because the scanning process is controlled by the vibrometer software that generates an instantaneous switching between measurement points.

The positioning of the voltage input exciting the electrical network is not obvious when we want to ensure an analogy with an external force that would be applied to 


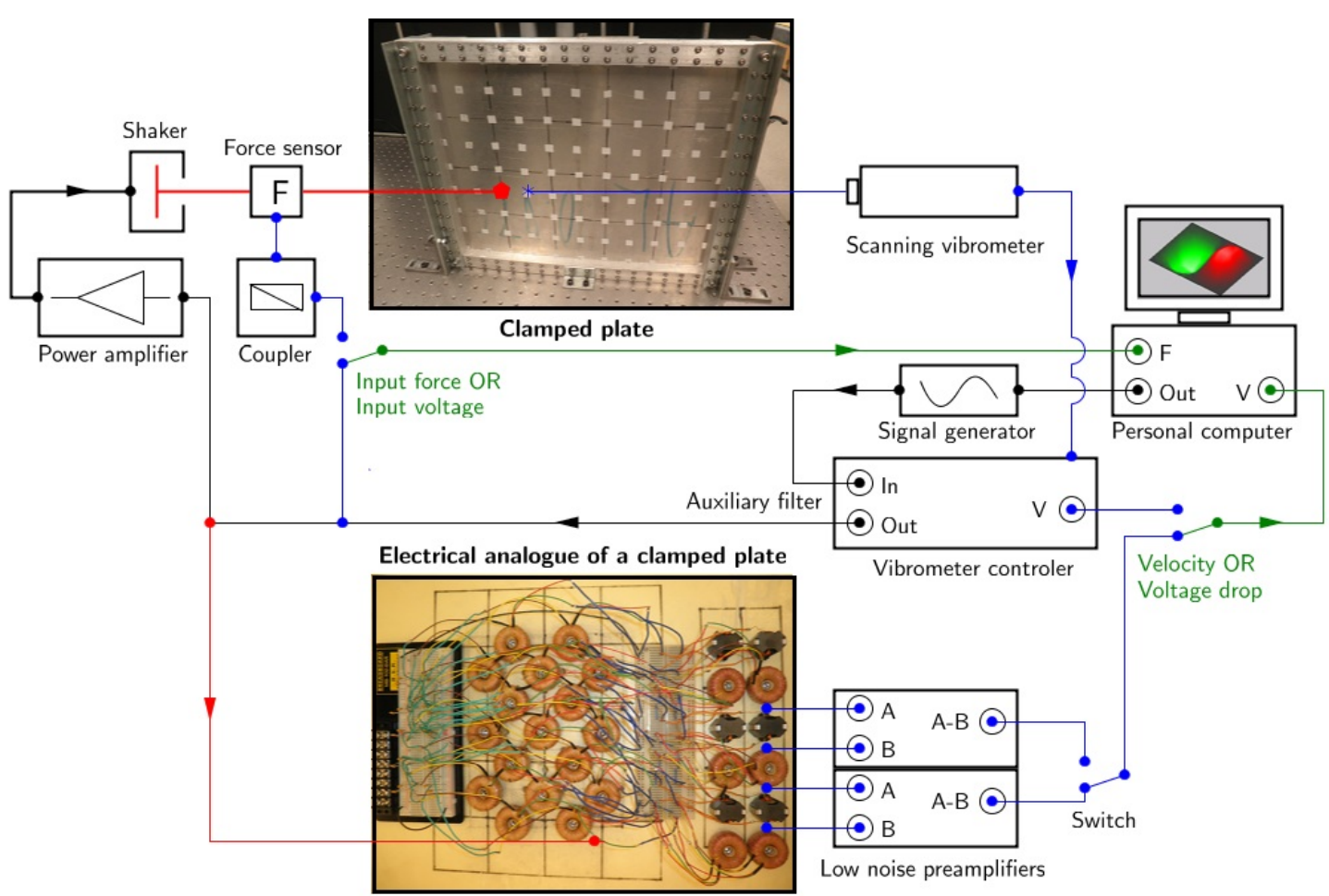

Figure 10. Experimental setup for the modal analysis of a clamped plate or for its analogous electrical network.

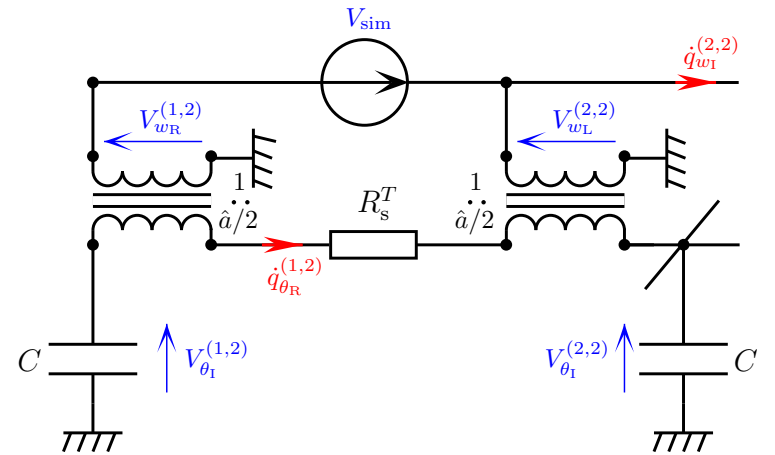

Figure 11. Theoretical excitation between two transformers.

a plate. Actually, the solution is given in Figure 6 for an excitation between the unit cells $(i-1, j)$ and $(i, j)$. For $i=$ $j=2$, electrical simplifications at the boundaries gives the electrical circuit in Figure 11, where appears the winding resistance $R_{\mathrm{s}}^{T}$ that replaces the inductance $L_{\theta}$ introduced in Figure 3 . The problem is that in our network all the pairs of $\hat{a} / 2: 1$ transformers have been replaced by $\hat{a}: 1$ transformers. It is thus not possible to directly generate a voltage $V_{\text {sim }}$ simulating a transverse force between the unit cells $(1,2)$ and $(2,2)$. Yet, we remark that Figure 11 gives

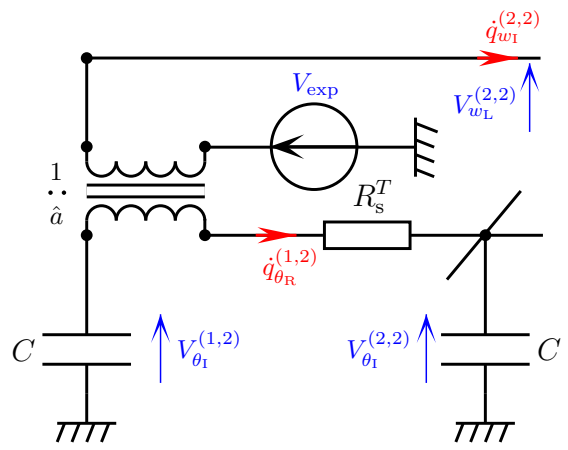

Figure 12. Simplified electrical circuit.

$$
V_{\theta_{\mathrm{I}}}^{(1,2)}-V_{\theta_{\mathrm{I}}}^{(2,2)}=\frac{\hat{a}}{2} V_{w_{\mathrm{R}}}^{(1,2)}+\mathrm{j} \omega R_{\mathrm{s}}^{T} \dot{q}_{\theta_{\mathrm{R}}}^{(1,2)}+\frac{\hat{a}}{2} V_{w_{\mathrm{L}}}^{(2,2)} .
$$

Furthermore, there is a relation between the voltage excitation $V_{\text {sim }}$ and the voltage across the transformers as

$$
V_{\text {sim }}=V_{w_{\mathrm{L}}}^{(2,2)}-V_{w_{\mathrm{R}}}^{(1,2)}
$$

Equations (30) and (31) thus lead to

$$
V_{w_{\mathrm{L}}}^{(2,2)}=\frac{1}{\hat{a}}\left(V_{\theta_{\mathrm{I}}}^{(1,2)}-\mathrm{j} \omega R_{\mathrm{s}}^{T} \dot{q}_{\theta_{\mathrm{R}}}^{(1,2)}-V_{\theta_{\mathrm{I}}}^{(2,2)}\right)+\frac{1}{2} V_{\mathrm{sim}},
$$


which is combined with

$$
\dot{q}_{w_{\mathrm{I}}}^{(2,2)}=\hat{a} \dot{q}_{\theta_{\mathrm{R}}}^{(1,2)}
$$

in order to give the simplified electrical scheme in Figure 12 where

$$
V_{\text {exp }}=\frac{1}{2} V_{\text {sim }} .
$$

All of this shows that placing a voltage excitation $V_{\exp }$ between the ground and the secondary winding of the $\hat{a}: 1$ transformer allows generating the analogue of a force between the $(1,2)$ and $(2,2)$ unit cells of the experimental network.

\section{Electrical distributions and frequency response functions}

The spatial distributions of the electrical current are represented in Figure 13 together with an electrical frequency response function representing the ratio of the voltage drop across the inductor in the $(2,2)$ unit cell to the excitation voltage $V_{\text {exp }}$. The six electrical resonances induced by the six inductors are identified between $50 \mathrm{~Hz}$ and $500 \mathrm{~Hz}$. Yet, they can be qualified as "smooth" when compared to the "sharp" resonances of a lightly damped mechanical system. The relatively low quality factor of the electrical resonances is mainly due to the winding resistance of the transformers. Nevertheless, the winding resistance does not necessarily need to be minimized because applications involving analogous piezoelectric control requires damping in the electrical network (Lossouarn et al. 2015, 2016). Concerning the spatial distributions of the currents around the resonances, it is seen that they represent a suitable approximation of the mode shapes of a clamped plate. This definitely validates the analogous electrical network which is able to reproduce the dynamics of a plate.

The experimental frequency response function is then compared to the result of the model developed in the previous section. The inductance values $L$ and $L_{\theta}$ appearing in the element matrices are replaced by their complex values including damping elements. Impedance calculations based on Figures 8 and 9 gives the equivalent inductance values

$$
\begin{aligned}
& L(\omega)=\frac{R_{\mathrm{p}}^{L} L_{0}}{R_{\mathrm{p}}^{L}+\mathrm{j} \omega L_{0}}+\frac{R_{\mathrm{s}}^{L}+R_{\mathrm{s}}^{T^{\star}}}{\mathrm{j} \omega} \\
& L_{\theta}(\omega)=\frac{R_{\mathrm{s}}^{T}}{\mathrm{j} \omega}
\end{aligned} .
$$

As stated previously, a voltage drop is measured across the inductor of the unit cell $(2,2)$. In our model, this corresponds to a voltage

$$
V_{\text {drop }}=-\omega^{2} L(\omega) q_{w_{\mathrm{I}}}^{(2,2)} .
$$

where $q_{w_{\mathrm{I}}}^{(2,2)}$ is the displacement of electric charges through the considered inductor. This charge displacement is defined as

$$
q_{w_{\mathrm{I}}}^{(2,2)}=q_{w_{\mathrm{L}}}^{(2,2)}+\frac{\hat{a}}{2} q_{\theta_{\mathrm{L}}}^{(2,2)},
$$

where $q_{w_{\mathrm{L}}}^{(2,2)}$ and $q_{\theta_{\mathrm{L}}}^{(2,2)}$ are computed from the model in equation (26). If $V_{\text {drop }}$ has been experimentally measured with an input $V_{\exp }=1$, a similar result should be obtained from a simulation with $V_{\text {sim }}=2$, as indicated by equation (34). Figure 13 finally shows that the simulation fits with the experimental frequency response function. Consequently, the proposed numerical model can help with the design of a suitable analogous network prior its actual implementation.

\section{Conclusions}

New plate electrical analogues has been defined for the purpose of damping structural vibration with a periodic array of piezoelectric patches. For a square plate unit cell, a finite difference method applied to the Kirchhoff-Love theory gives a discrete model that is converted into its direct electrical analogue. The analogues of clamped or simply-supported boundary conditions only require openor short-circuited electrical ports along the edges of the network. Yet, free boundary conditions are more difficult to implement with the proposed topology. In any case, the electrical network can be modeled from an assembly of element matrices that are built from the constitutive discrete equations of an electrical unit cell. When increasing the number of unit cells, the analogous network tends to the model of the continuous structure. It is then shown that the proposed electrical analogue represents a suitable 


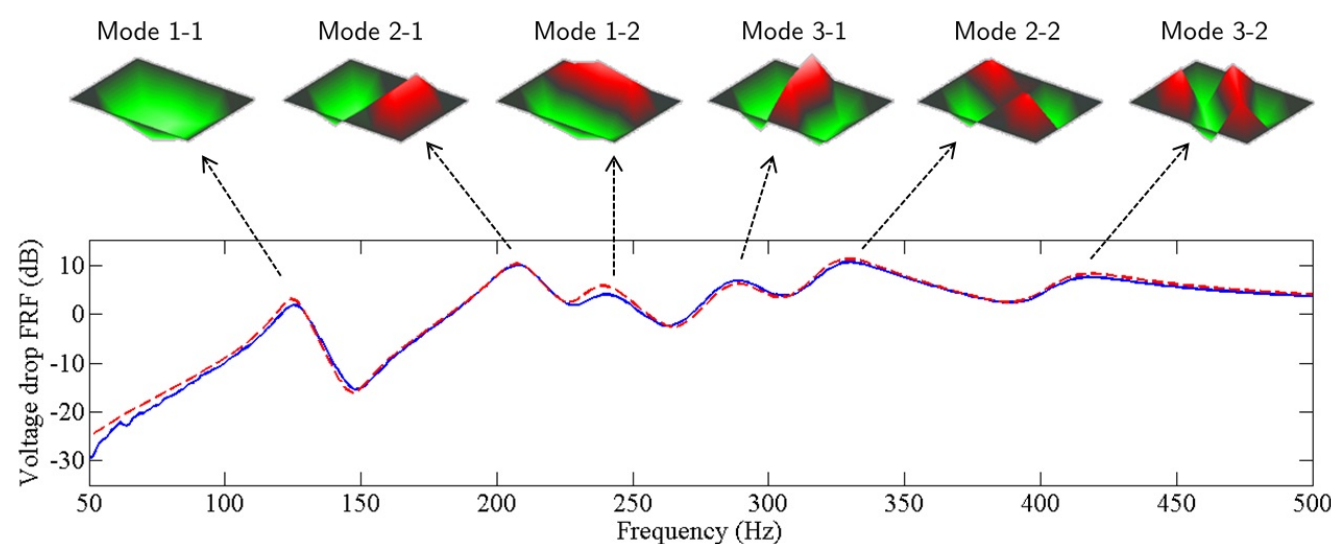

Figure 13. Experimental distributions of the electrical current with respect to the voltage frequency response functions - $(-)$ experiment, $(--)$ simulation.

approximation of a plate, when it offers more than 10 unit cells per wavelength.

Considering the practical implementation of a passive electrical analogue, guidelines are given for the design of the electrical transformers which require sufficient magnetizing inductance. After assembling the electrical components, the analogous network is validated through a modal analysis procedure similar to what is usually performed for a mechanical structure. Electrical plate-like mode shapes are observed experimentally when looking at the distribution of the electrical current. Furthermore, the numerical simulations of the network are in good agreement with the experimental results, which shows the interest of the proposed model for preliminary evaluations of the electrical responses. In the end, it becomes possible to design efficient analogous controllers for subsequent passive vibration damping of plates. Since the proposed electrical network has been successfully coupled to a clamped plate, future work will be dedicated to the design of an analogous network for vibration damping of a simplysupported plate.

\section{Appendix. Element "mass" and "stiffness" matrices}

From the unit cell in Figure 3, the impedance of the capacitor $C$ induces

$$
V_{\theta_{\mathrm{I}}}^{\star}=\frac{1}{\mathrm{j} \omega C}\left(\dot{q}_{\theta_{\mathrm{L}}}^{\star}-\dot{q}_{\theta_{\mathrm{R}}}^{\star}+\dot{q}_{\theta_{\mathrm{B}}}^{\star}-\dot{q}_{\theta_{\mathrm{T}}}^{\star}\right)
$$

and the inductor $L$ leads to

$$
V_{w_{\mathrm{L}}}-\left(V_{w_{\mathrm{T}}}-V_{w_{\mathrm{B}}}\right)-V_{w_{\mathrm{R}}}=\mathrm{j} \omega L \dot{q}_{w_{\mathrm{I}}}^{\star}
$$

Furthermore, the transformers of ratio $\hat{a} / 2$ gives

$$
\begin{aligned}
& V_{\theta_{\mathrm{L}}}^{\star}-V_{\theta_{\mathrm{I}}}^{\star}=\frac{\hat{a}}{2} V_{w_{\mathrm{L}}} \\
& V_{\theta_{\mathrm{I}}}^{\star}-V_{\theta_{\mathrm{R}}}^{\star}=\frac{\hat{a}}{2} V_{w_{\mathrm{R}}} \\
& V_{\theta_{\mathrm{B}}}^{\star}-V_{\theta_{\mathrm{I}}}^{\star}=\frac{\hat{a}}{2} V_{w_{\mathrm{B}}} \\
& V_{\theta_{\mathrm{I}}}^{\star}-V_{\theta_{\mathrm{T}}}^{\star}=\frac{\hat{a}}{2} V_{w_{\mathrm{T}}} \\
& \frac{\hat{a}}{2} \dot{q}_{\theta_{\mathrm{L}}}^{\star}=\dot{q}_{w_{\mathrm{I}}}^{\star}-\dot{q}_{w_{\mathrm{L}}} \\
& \frac{\hat{a}}{2} \dot{q}_{\theta_{\mathrm{R}}}^{\star}=\dot{q}_{w_{\mathrm{R}}}-\dot{q}_{w_{\mathrm{I}}}^{\star} \\
& \frac{\hat{a}}{2} \dot{q}_{\theta_{\mathrm{B}}}^{\star}=\dot{q}_{w_{\mathrm{I}}}^{\star}-\dot{q}_{w_{\mathrm{B}}}^{\star} \\
& \frac{\hat{a}}{2} \dot{q}_{\theta_{\mathrm{T}}}^{\star}=\dot{q}_{w_{\mathrm{T}}}-\dot{q}_{w_{\mathrm{I}}}^{\star}
\end{aligned}
$$

At last, the additional components $L_{\theta} / 2$ and $C_{0} / 2$ induce

$$
\begin{aligned}
& V_{\theta_{\mathrm{L}}}-V_{\theta_{\mathrm{L}}}^{\star}=\mathrm{j} \omega \frac{L_{\theta}}{2} \dot{q}_{\theta_{\mathrm{L}}}^{\star} \\
& V_{\theta_{\mathrm{R}}}^{\star}-V_{\theta_{\mathrm{R}}}=\mathrm{j} \omega \frac{L_{\theta}}{2} \dot{q}_{\theta_{\mathrm{R}}}^{\star} \\
& V_{\theta_{\mathrm{B}}}-V_{\theta_{\mathrm{B}}}^{\star}=\mathrm{j} \omega \frac{L_{\theta}}{2} \dot{q}_{\theta_{\mathrm{B}}}^{\star} \\
& V_{\theta_{\mathrm{T}}}^{\star}-V_{\theta_{\mathrm{T}}}=\mathrm{j} \omega \frac{L_{\theta}}{2} \dot{q}_{\theta_{\mathrm{T}}}^{\star} \\
& V_{\theta_{\mathrm{L}}}=\frac{2}{\mathrm{j} \omega C_{0}}\left(\dot{q}_{\theta_{\mathrm{L}}}-\dot{q}_{\theta_{\mathrm{L}}}^{\star}\right) \\
& V_{\theta_{\mathrm{R}}}=\frac{2}{\mathrm{j} \omega C_{0}}\left(\dot{q}_{\theta_{\mathrm{R}}}^{\star}-\dot{q}_{\theta_{\mathrm{R}}}\right) \\
& V_{\theta_{\mathrm{B}}}=\frac{2}{\mathrm{j} \omega C_{0}}\left(\dot{q}_{\theta_{\mathrm{B}}}-\dot{q}_{\theta_{\mathrm{B}}}^{\star}\right) \\
& V_{\theta_{\mathrm{T}}}=\frac{2}{\mathrm{j} \omega C_{0}}\left(\dot{q}_{\theta_{\mathrm{T}}}^{\star}-\dot{q}_{\theta_{\mathrm{T}}}\right)
\end{aligned}
$$

The internal variables denoted by the $\star$ superscript are eliminated from the system of equations with a symbolic solver, which is then used to express the remaining voltage variables as linear combinations of the electric 


$$
\boldsymbol{K}_{\boldsymbol{e}}^{\mathrm{e}}=\frac{1}{\hat{a}^{2} C_{0}}\left[\begin{array}{cccccccc}
6 & 3 \hat{a} & -2 & -\hat{a} & -2 & \hat{a} & -2 & \hat{a} \\
3 \hat{a} & \frac{\hat{a}^{2}}{2} \frac{3 C+8 C_{0}}{C+2 C_{0}} & -\hat{a} & -\frac{\hat{a}^{2}}{2} \frac{C}{C+2 C_{0}} & -\hat{a} & \frac{\hat{a}^{2}}{2} \frac{C}{C+2 C_{0}} & -\hat{a} & \frac{\hat{a}^{2}}{2} \frac{C}{C+2 C_{0}} \\
-2 & -\hat{a} & 6 & 3 \hat{a} & -2 & \hat{a} & -2 & \hat{a} \\
-\hat{a} & -\frac{\hat{a}^{2}}{2} \frac{C}{C+2 C_{0}} & 3 \hat{a} & \frac{\hat{a}^{2}}{2} \frac{3 C+8 C_{0}}{C+2 C_{0}} & -\hat{a} & \frac{\hat{a}^{2}}{2} \frac{C}{C+2 C_{0}} & -\hat{a} & \frac{\hat{a}^{2}}{2} \frac{C}{C+2 C_{0}} \\
-2 & -\hat{a} & -2 & -\hat{a} & 6 & -3 \hat{a} & -2 & \hat{a} \\
\hat{a} & \frac{\hat{a}^{2}}{2} \frac{C}{C+2 C_{0}} & \hat{a} & \frac{\hat{a}^{2}}{2} \frac{C}{C+2 C_{0}} & -3 \hat{a} & \frac{\hat{a}^{2}}{2} \frac{3 C+8 C_{0}}{C+2 C_{0}} & \hat{a} & -\frac{\hat{a}^{2}}{2} \frac{C}{C+2 C_{0}} \\
-2 & -\hat{a} & -2 & -\hat{a} & -2 & \frac{\hat{a}}{2} & 6 & -3 \hat{a} \\
\hat{a} & \frac{\hat{a}^{2}}{2} \frac{C}{C+2 C_{0}} & \hat{a} & \frac{\hat{a}^{2}}{2} \frac{C}{C+2 C_{0}} & \hat{a} & -\frac{\hat{a}^{2}}{2} \frac{C}{C+2 C_{0}} & -3 \hat{a} & \frac{\hat{a}^{2}}{2} \frac{3 C+8 C_{0}}{C+2 C_{0}}
\end{array}\right]
$$

charge displacement variables. This gives the elements of the "dynamic stiffness matrix" $\boldsymbol{D}_{e}^{\mathrm{e}}$ in equation (18) and from equation (21) it is found the "stiffness" matrix in equation (42). Finally, from equation (22) and by setting $C_{0}=0$, the "mass" matrices in equation (23) are

$$
\boldsymbol{M}_{\boldsymbol{e}}^{\boldsymbol{L}}=\frac{L}{16}\left[\begin{array}{cccccccc}
1 & \frac{\hat{a}}{2} & 1 & \frac{\hat{a}}{2} & 1 & -\frac{\hat{a}}{2} & 1 & -\frac{\hat{a}}{2} \\
\frac{\hat{a}}{2} & \frac{\hat{a}^{2}}{4} & \frac{\hat{a}}{2} & \frac{\hat{a}^{2}}{4} & \frac{\hat{a}}{2} & -\frac{\hat{a}^{2}}{4} & \frac{\hat{a}}{2} & -\frac{\hat{a}^{2}}{4} \\
1 & \frac{\hat{a}}{2} & 1 & \frac{\hat{a}}{2} & 1 & -\frac{\hat{a}}{2} & 1 & -\frac{\hat{a}}{2} \\
\frac{\hat{a}}{2} & \frac{\hat{a}^{2}}{4} & \frac{\hat{a}}{2} & \frac{\hat{a}^{2}}{4} & \frac{\hat{a}}{2} & -\frac{\hat{a}^{2}}{4} & \frac{\hat{a}}{2} & -\frac{\hat{a}^{2}}{4} \\
1 & \frac{\hat{a}}{2} & 1 & \frac{\hat{a}}{2} & 1 & -\frac{\hat{a}}{2} & 1 & -\frac{\hat{a}}{2} \\
-\frac{\hat{a}}{2} & -\frac{\hat{a}^{2}}{4} & -\frac{\hat{a}}{2} & -\frac{\hat{a}^{2}}{4} & -\frac{\hat{a}}{2} & \frac{\hat{a}^{2}}{4} & -\frac{\hat{a}}{2} & \frac{\hat{a}^{2}}{4} \\
1 & \frac{\hat{a}}{2} & 1 & \frac{\hat{a}}{2} & 1 & -\frac{\hat{a}}{2} & 1 & -\frac{\hat{a}}{2} \\
-\frac{\hat{a}}{2} & -\frac{\hat{a}^{2}}{4} & -\frac{\hat{a}}{2} & -\frac{\hat{a}^{2}}{4} & -\frac{\hat{a}}{2} & \frac{\hat{a}^{2}}{4} & -\frac{\hat{a}}{2} & \frac{\hat{a}^{2}}{4}
\end{array}\right]
$$

and

$$
\boldsymbol{M}_{\boldsymbol{e}}^{\boldsymbol{L}_{\boldsymbol{\theta}}}=\frac{L_{\theta}}{2 a^{2}}\left[\begin{array}{cccccccc}
3 & 0 & -1 & 0 & -1 & 0 & -1 & 0 \\
0 & \frac{\hat{a}^{2}}{4} & 0 & \frac{\hat{a}^{2}}{4} & 0 & -\frac{\hat{a}^{2}}{4} & 0 & -\frac{\hat{a}^{2}}{4} \\
-1 & 0 & 3 & 0 & -1 & 0 & -1 & 0 \\
0 & \frac{\hat{a}^{2}}{4} & 0 & \frac{\hat{a}^{2}}{4} & 0 & -\frac{\hat{a}^{2}}{4} & 0 & -\frac{\hat{a}^{2}}{4} \\
-1 & 0 & -1 & 0 & 3 & 0 & -1 & 0 \\
0 & -\frac{\hat{a}^{2}}{4} & 0 & -\frac{\hat{a}^{2}}{4} & 0 & \frac{\hat{a}^{2}}{4} & 0 & \frac{\hat{a}^{2}}{4} \\
-1 & 0 & -1 & 0 & -1 & 0 & 3 & 0 \\
0 & -\frac{\hat{a}^{2}}{4} & 0 & -\frac{\hat{a}^{2}}{4} & 0 & \frac{\hat{a}^{2}}{4} & 0 & \frac{\hat{a}^{2}}{4}
\end{array}\right] .
$$

\section{Funding}

This work was funded by the French Ministry of National Education, Higher Education and Research through a three year scholarship for doctoral studies related to structural damping with piezoelectric devices.

\section{Acknowledgements}

The authors want to thank the Fulbright Program, which made possible a nine-month research visit at the Georgia Institute of Technology.

\section{Declaration of conflicting interests}

The Authors declare that there is no conflict of interest with respect to the research, authorship, and/or publication of this article.

\section{References}

Airoldi L and Ruzzene M (2011) Wave propagation control in beams through periodic multi-branch shunts. Journal of Intelligent Material Systems and Structures 22(14): 1567-1579. DOI:10.1177/1045389X11408372. URL http://jim. sagepub.com/content/22/14/1567.abstract.

Alessandroni S, Andreaus U, Dell'Isola F and Porfiri M (2004) Piezo-electromechanical (PEM) Kirchhoff-Love plates. European Journal of Mechanics - A/Solids 23(4): 689-702. DOI:http://dx.doi.org/10.1016/j.euromechsol.2004.03.003.

URL http: / / www. sciencedirect.com/science/ article/pii/s0997753804000476.

Alessandroni S, Andreaus U, Dell'Isola F and Porfiri M (2005) A passive electric controller for multimodal vibrations of thin plates. Computers \& Structures 83(15-16): 1236-1250. DOI:http://dx.doi.org/10.1016/j.compstruc.2004.08.028.

URL http: //www. sciencedirect.com/science/ article/pii/s0045794905000283.

Alessandroni S, Dell'Isola F and Porfiri M (2002) A revival of electric analogs for vibrating mechanical systems aimed to their efficient control by PZT actuators. International Journal of Solids and Structures 39(20): 5295-5324. DOI: http://dx.doi.org/10.1016/S0020-7683(02)00402-X. URL 
http://www.sciencedirect.com/science/ article/pii/S002076830200402X.

Andreaus U, Dell'Isola F and Porfiri M (2004) Piezoelectric passive distributed controllers for beam flexural vibrations. Journal of Vibration and Control 10(5): 625-659. DOI:10. 1177/1077546304038224. URL http: / / jvc. sagepub . com/content/10/5/625. abstract.

Barnoski R and Freberg C (1966) Passive-element analog circuits for three-dimensional elasticity. Journal of Engineering for Industry 88(1): 8-16.

Benscoter SU and MacNeal RH (1952a) Equivalent-plate theory for a straight multicell wing. Technical report, NACA. URL http://naca.central.cranfield. ac.uk/report.php?NID=4942.

Benscoter SU and MacNeal RH (1952b) Introduction to electricalcircuit analogies for beam analysis. Technical report, NACA. URL http://naca.central.cranfield. ac.uk/report.php?NID=4941.

Beranek LL (1954) Acoustics. McGraw-Hill.

Bloch A (1945) Electromechanical analogies and their use for the analysis of mechanical and electromechanical systems. Journal of the Institution of Electrical Engineers - Part I: General 92(52): 157-169. DOI:10.1049/ji-1.1945.0039.

Bush V (1934) Structural analysis by electric circuit analogies. Journal of the Franklin Institute 217(3): 289-329. DOI: http://dx.doi.org/10.1016/S0016-0032(34)90575-5. URL http://www.sciencedirect.com/science/ article/pii/s0016003234905755.

Carter G and Kron G (1944) Network analyzer solution of the equivalent circuits for elastic structures. Journal of the Franklin Institute 238(6): 443-452. DOI: http://dx.doi.org/10.1016/S0016-0032(44)91081-1. URL http://www.sciencedirect.com/science/ article/pii/s0016003244910811.

Cho YS, Pak Y, Han CS and Ha SK (2000) Five-port equivalent electric circuit of piezoelectric bimorph beam. Sensors and Actuators A: Physical 84(1-2): 140-148. DOI: http://dx.doi.org/10.1016/S0924-4247(99)00231-9. URL http://www.sciencedirect.com/science/ article/pii/s0924424799002319.

Dell'Isola F, Maurini C and Porfiri M (2004) Passive damping of beam vibrations through distributed electric networks and piezoelectric transducers: prototype design and experimental validation. Smart Materials and Structures 13(2): 299-308. URL http://stacks.iop.org/0964-1726/13/i= $2 / a=008$.

Dell'Isola F, Porfiri M and Vidoli S (2003) Piezoelectromechanical (PEM) structures: passive vibration control using distributed piezoelectric transducers. Comptes Rendus Mécanique 331(1): 69-76. DOI: http://dx.doi.org/10.1016/S1631-0721(03)00022-6. URL

http://www.sciencedirect.com/science/ article/pii/s1631072103000226.

Firestone FA (1933) A new analogy between mechanical and electrical systems. The Journal of the Acoustical Society of America 4(3): 249-267. DOI:http://dx.doi.org/10.1121/1.1915605. URL http://scitation.aip.org/content/asa/ journal/jasa/4/3/10.1121/1.1915605.

Hollkamp JJ (1994) Multimodal passive vibration suppression with piezoelectric materials and resonant shunts. Journal of Intelligent Material Systems and Structures 5(1): 49-57. DOI:10.1177/1045389X9400500106. URL http:// jim. sagepub.com/content/5/1/49. abstract.

Kron G (1944) Tensorial analysis and equivalent circuits of elastic structures. Journal of the Franklin Institute 238(6): 399-442. DOI:http://dx.doi.org/10.1016/S0016-0032(44) 91080-X. URL http://www.sciencedirect.com/ science/article/pii/s001600324491080x.

Kron G (1945) Numerical solution of ordinary and partial differential equations by means of equivalent circuits. Journal of Applied Physics 16(3): 172186. DOI:http://dx.doi.org/10.1063/1.1707568. URL http://scitation.aip.org/content/aip/ journal/jap/16/3/10.1063/1.1707568.

Kron G (1948) Electric circuit models of partial differential equations. Electrical Engineering 67(7): 672-684. DOI: 10.1109/EE.1948.6444220.

Lallart M, Magnet C, Richard C, Lefeuvre E, Petit L, Guyomar D and Bouillault F (2008) New synchronized switch damping methods using dual transformations. Sensors and Actuators A: Physical 143(2): 302-314. DOI:http://dx.doi.org/10.1016/j.sna.2007.12.001. URL http: //www. sciencedirect.com/science/ article/pii/s0924424707008874. 
Lossouarn B, Deü JF and Aucejo M (2015) Multimodal vibration damping of a beam with a periodic array of piezoelectric patches connected to a passive electrical network. Smart Materials and Structures 24(11): 115037. URL http://stacks.iop.org/0964-1726/24/i= $11 / \mathrm{a}=115037$.

Lossouarn B, Deü JF, Aucejo M and Cunefare KA (2016) Multimodal vibration damping of a plate by piezoelectric coupling to its analogous electrical network. Smart Materials and Structures 25(11): 115042. URL http://stacks . iop.org/0964-1726/25/i=11/a=115042.

MacNeal RH (1949) The solution of partial differential equations by means of electrical networks. $\mathrm{PhD}$ Thesis. URL http://resolver.caltech.edu/ CaltechETD : etd-04282004-143609.

MacNeal RH (1951) The solution of aeroelastic problems by means of electrical analogies. Journal of the Aeronautical Sciences .

MacNeal RH (1954) Electrical analogies for stiffened shells with flexible rings. Technical report, NACA. URL

http://naca.central.cranfield.ac.uk/ report .php?NID=5940.

Maurini C (2005) Piezoelectric composites for distributed passive electric control: beam modelling, modal analysis, and experimental implementation. $\mathrm{PhD}$ Thesis, Université Pierre et Marie Curie - Paris VI ; Università degli studi di Roma I. URL https://tel.archives-ouvertes.fr/ tel-00011252.

Paganelli RP, Romani A, Golfarelli A, Magi M, Sangiorgi E and Tartagni M (2010) Modeling and characterization of piezoelectric transducers by means of scattering parameters. Part I: Theory. Sensors and Actuators A: Physical 160(12): 9-18. DOI:http://dx.doi.org/10.1016/j.sna.2010. 03.006. URL http://www.sciencedirect.com/ science/article/pii/s0924424710001093.

Panella M, Paschero M and Frattale Mascioli FM (2005) Optimised RC-active synthesis of PEM networks. Electronics Letters 41(19): 1041-1043.

Paschero M, Panella M and Frattale Mascioli FM (2006) Stability analysis of optimal PEM networks. Electronics Letters 42(17): 961-962. DOI:10.1049/el:20062114.
Porfiri M, Dell'Isola F and Frattale Mascioli FM (2004) Circuit analog of a beam and its application to multimodal vibration damping, using piezoelectric transducers. International Journal of Circuit Theory and Applications 32(4): 167-198. DOI:10.1002/cta.273. URL http://dx.doi.org/10. $1002 /$ cta. 273.

Timoshenko SP (1940) Theory of plates and shells. McGraw-Hill. Valis T, von Flotow A and Hagood N (1994) An acousticelectromagnetic piezoelectric waveguide coupler. Journal of Sound and Vibration 178(5): 669-680. DOI:http://dx.doi.org/10.1006/jsvi.1994.1514.

URL

http://www.sciencedirect.com/science/

article/pii/s0022460X84715148.

Vidoli S and Dell'Isola F (2001) Vibration control in plates by uniformly distributed PZT actuators interconnected via electric networks. European Journal of Mechanics - A/Solids 20(3): 435-456. DOI:http://dx.doi.org/10.1016/S0997-7538(01) 01144-5. URL http://WWW.sciencedirect.com/ science/article/pii/s0997753801011445.

Wu SY (1998) Method for multiple mode piezoelectric shunting with single PZT transducer for vibration control. Journal of Intelligent Material Systems and Structures 9(12): 991-998. DOI:10.1177/1045389X9800901204. URL http://jim. sagepub.com/content/9/12/991. abstract. 